\title{
Morbidity and Mortality: A Case Report of Metastatic Bone Disease
}

\author{
Muhammad Siddique Pir ${ }^{1}$, Jumana Jaloudi ${ }^{1}$, Mariam Mir ${ }^{1}$, Najam Saqib ${ }^{1}$, Douglas Klamp ${ }^{1}$ \\ 1. Department of Internal Medicine, The Wright Center for Graduate Medical Education, Scranton, USA
}

Corresponding author: Muhammad Siddique Pir, pirsiddiq@gmail.com

\begin{abstract}
Metastatic prostate cancer and multiple myeloma (MM) usually present with bone lesions, posing a diagnostic challenge in males presenting in late stages. In this case report, an 86-year-old male who had not seen a physician in over 30 years presented with complaints of hip pain and progressive difficulty in walking for three weeks. Outpatient X-ray of the right hip showed multiple lytic bone lesions, raising suspicion of MM. Other laboratory tests revealed elevated serum calcium and elevated prostate-specific antigen (PSA), supporting a diagnosis of prostate cancer. The patient was admitted for further workup. Magnetic resonance imaging (MRI) of the spine showed diffuse metastatic disease throughout the spine as well as pelvis with multilevel central canal and neuro-foraminal narrowing due to degenerative changes. Central canal narrowing at L1-L2 due to tumor involvement could not be ruled out on MRI.
\end{abstract}

Subsequently, urology was consulted and the patient was taken to the operating room for prostate biopsy and possible bilateral orchiectomy. Two intraoperative prostate biopsies were negative for malignancy but patient underwent bilateral orchiectomy due to high clinical suspicion for prostate cancer. Bone lesions in the pelvis were so extensive that orthopedic surgeons recommended complete non-weight bearing as the risk of fracture with weight bearing was thought to be very high. Eventually, laboratory workup for MM came out to be positive. Radiation oncologist recommended radiation therapy; however, at this point, the patient refused further intervention. He opted for palliative care. Consequently, a bone marrow biopsy could not be obtained for a definitive diagnosis of MM. The patient was eventually discharged to a nursing home for hospice care.

This case sheds light on the importance of preventative care in routine outpatient setting, which can often screen, identify, and detect malignancies at earlier stages. It also signifies the importance of an interdisciplinary approach and precise knowledge in differentiating and diagnosing such malignancies. In our patient's case, his extensive bone disease precluded his ability to be weight bearing which is an uncommon finding only seen in extensive metastatic bone disease. A definitive diagnosis is warranted to guide appropriate management.

Received 06/09/2018 Review began $07 / 05 / 2018$ Review ended 12/23/2018 Published 12/27/2018

\section{(C) Copyright 2018} Pir et al. This is an open access article distributed under the terms of the Creative Commons Attribution License CC-BY 3.0., which permits unrestricted use, distribution, and reproduction in any medium, provided the original author and source are credited.
Categories: Internal Medicine, Urology, Oncology

Keywords: prostate cancer, multiple myeloma, lytic bone lesions, screening, metastatic bone disease, hypercalcemia, prostate-specific antigen

\section{Introduction}

Metastatic prostate cancer and multiple myeloma (MM) both commonly lead to bone involvement. In advance stages, it may become challenging to make a clinical diagnosis due to overlapping features. This case report highlights such a case with several features in support of each diagnosis. This case also sheds light on the importance of regular primary care follow up visits for preventive care. Prostate-specific antigen (PSA)-based prostate cancer screening (even though somewhat controversial) may, in fact, have a very consequential impact in such cases.

\section{Case Presentation}

An 86-year-old male with no known past medical history presented to his primary care physician with complaints of sudden onset of right hip pain and progressive difficulty in walking for three weeks. He did not follow-up with any physician for over 30 years. He reported severe pain that was exacerbated by any physical activity and movement. His pain was minimally relieved with rest and radiated to bilateral lower extremities. Outpatient chest and right hip X-rays revealed multiple lytic lesions, suggestive of metastatic disease. The patient was admitted to the hospital for further workup. X-rays of the pelvis revealed multiple lytic lesions throughout the entire pelvis and bilateral femurs (Figures 1-3). Laboratory tests were significant for the following: hemoglobin level of $9 \mathrm{~g} / \mathrm{dl}$, creatinine $2.8 \mathrm{mg} / \mathrm{dl}$, calcium $13.5 \mathrm{mg} / \mathrm{dl}$, albumin $2.7 \mathrm{~g} / \mathrm{dl}$, total serum protein $9.6 \mathrm{~g} / \mathrm{dl}$, globulin $7.1 \mathrm{~g} / \mathrm{dl}$, albumin to globulin ratio 0.3 , free PSA $6.9 \mathrm{ng} / \mathrm{mL}$ and total PSA 78.0 $\mathrm{ng} / \mathrm{mL}$. Urinalysis showed proteinuria and hematuria. Magnetic resonance imaging (MRI) of the spine revealed diffuse metastatic disease (Figure 4). A bone scan showed multiple foci of increased activity throughout bilateral ribs, spinous processes of the lumbar spine, bilateral shoulders, left iliac wing and left acetabulum consistent with metastatic disease (Figure 5). At this point, the working diagnoses included MM 


\section{Cureus}

and metastatic prostate cancer. Urology proceeded with a prostate biopsy. Despite negative intra-operative frozen sections for prostate cancer, a bilateral orchiectomy was performed due to high clinical suspicion of prostate cancer. The aim of this procedure was to achieve early androgen deprivation to prevent spinal cord compression. The patient underwent the procedure without any complications. He was started on steroids for suspected spinal cord compression. Intravenous fluid hydration and zoledronic acid were administered for hypercalcemia. Subsequent laboratory tests showed improvement in calcium level. Serum creatinine level remained elevated between $2 \mathrm{mg} / \mathrm{dl}$ and $3 \mathrm{mg} / \mathrm{dl}$. MM workup including serum protein electrophoresis, serum, and urine free kappa and lambda light chains were supportive of a diagnosis of MM: free kappa serum level $19.7 \mathrm{mg} / \mathrm{dl}$, free lambda serum level $3099 \mathrm{mg} / \mathrm{dl}$, free kappa urine $164 \mathrm{mg} / \mathrm{L}$, free lambda urine $1500 \mathrm{mg} / \mathrm{L}$, and free kappa/lambda urine ratio 0.11. Immunofixation was interpreted as monoclonal IgG lambda present. According to the Revised International Myeloma Working Group Diagnostic Criteria for MM, tissue evidence of $>10 \%$ of bone or extramedullary plasmacytoma is necessary for a definitive diagnosis of MM. It was recommended by orthopedic surgeons that the patient be on bed rest indefinitely due to high fracture risk as a result of his bone frailty. The patient was given the option to proceed with bone marrow biopsy and appropriate treatment based on results. Radiation therapy to help with the pain was also recommended. After extensive discussion about all diagnostic, therapeutic and palliative options patient opted for comfort measures only. He did not want to pursue further diagnostic workup as his quality of life was expected to be severely diminished. The patient was eventually discharged to a nursing home for hospice care.

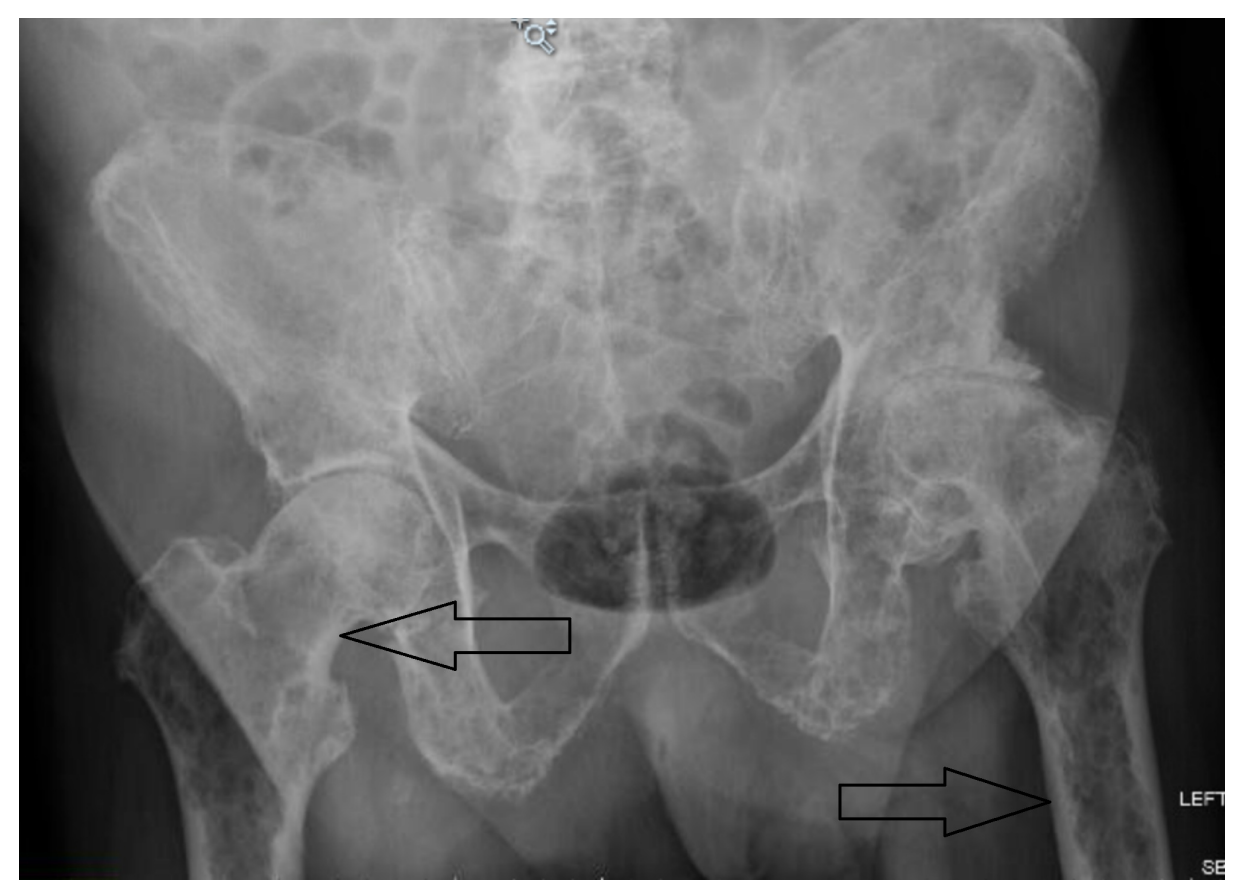

FIGURE 1: X-ray of the pelvis

Showing extensive lytic bone lesions marked by arrows. 


\section{Cureus}

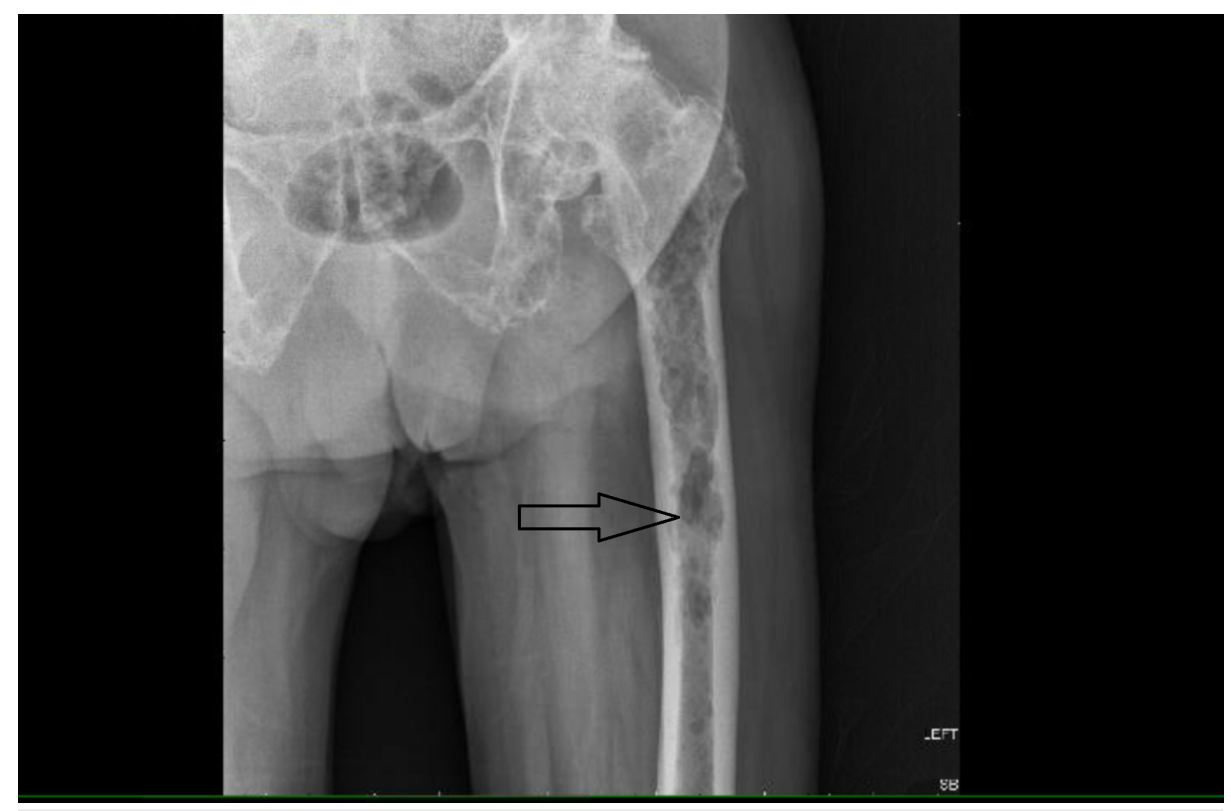

FIGURE 2: X-ray of the femur

Showing multiple lytic lesions; arrow points towards one big lytic lesion.

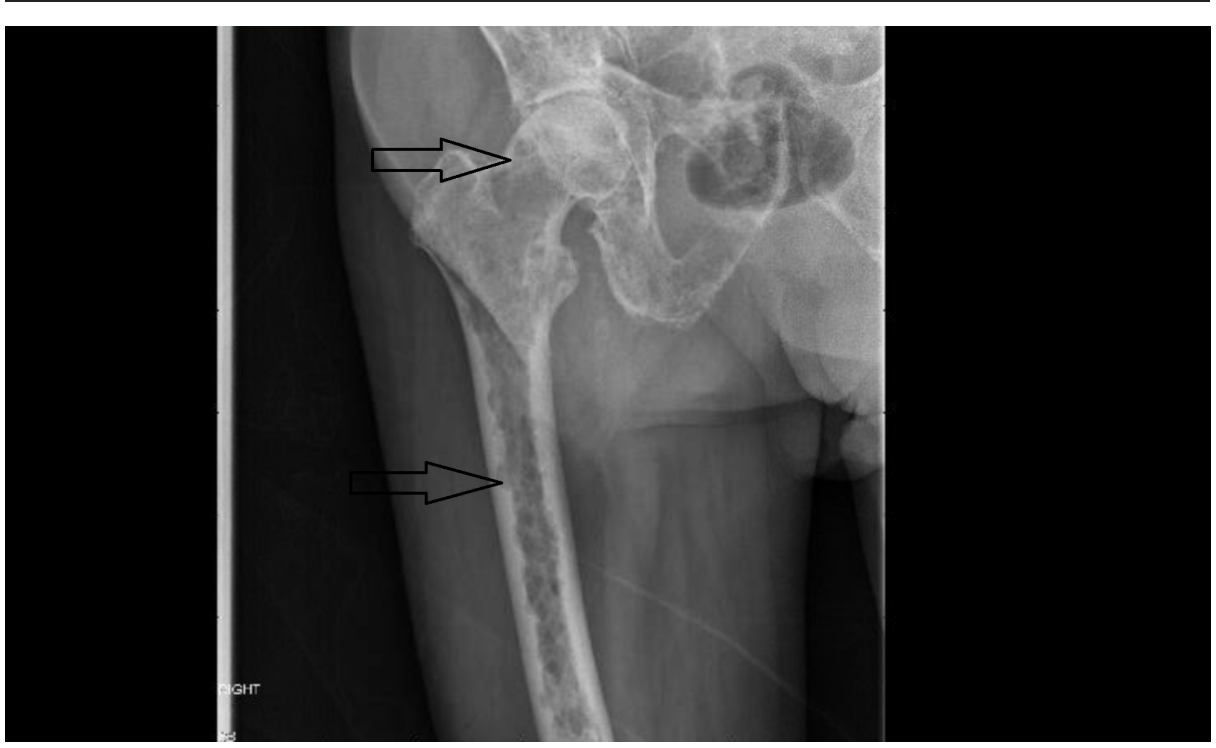

FIGURE 3: X-ray of the femur

Showing multiple lytic lesions marked by arrows. 


\section{Cureus}

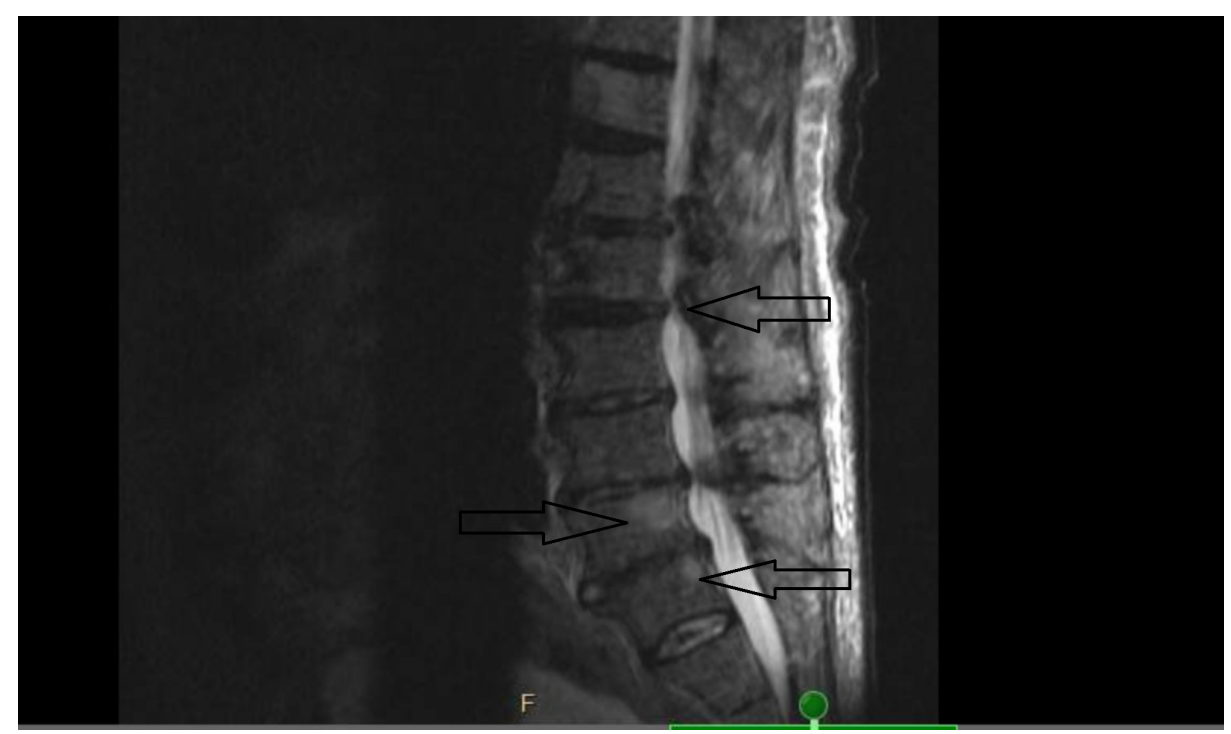

\section{FIGURE 4: Magnetic resonance imaging (MRI) of the lumbar spine}

Showing indeterminate cord compression (marked by an arrow) and multiple bone lesions, two of them marked by arrows.

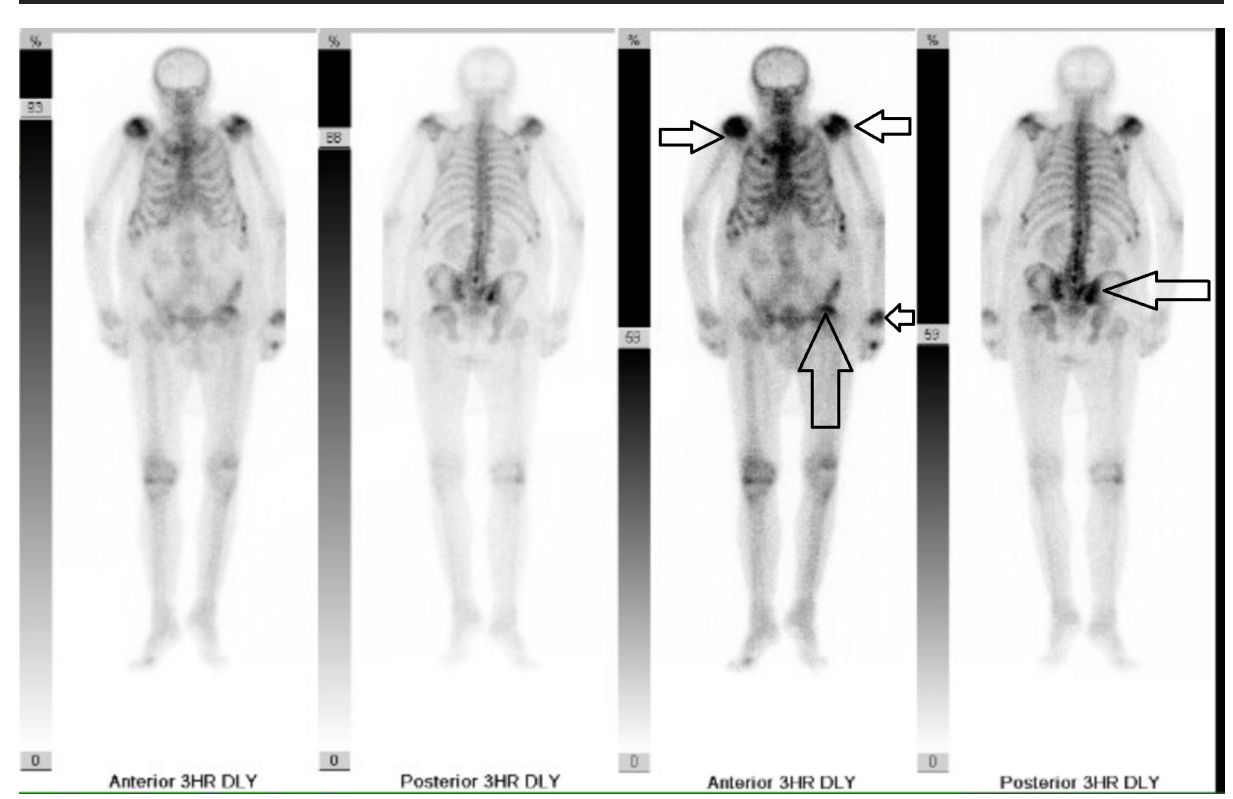

\section{FIGURE 5: Bone scan}

Showing metastatic disease in multiple areas including the shoulders, left wrist, left side of the pelvis, ribs, and sternum.

\section{Discussion}

Advanced prostate cancer and MM are two very distinct malignancies with differing underlying pathogenesis and management; however, they share several overlapping clinical features, especially in late or advanced disease, which may pose a diagnostic challenge for clinicians [1-2]. MM is typically associated with lytic bone lesions while prostate cancer typically presents with osteoblastic lesions; however, prostate cancer may present as osteolytic bone lesions as well [3-4]. Moreover, prostate cancer is the most common malignancy in males, especially in the elderly, making it a likely diagnosis in our case at initial presentation.

Early androgen deprivation therapy with bilateral orchiectomy or with chemical castration is recommended for suspected metastatic prostate cancer [5] especially in case of suspected or actual spinal cord compression. A study published in the South African Journal of Surgery in 2014 suggested that the diagnosis of advanced prostate cancer may be established based on clinical features and elevated PSA alone without 
tissue biopsy and androgen deprivation therapy may be initiated [6]. Our patient presented with evidence suggestive of impending cord compression, therefore, early aggressive therapy was suggested for androgendeprivation and surgical castration was thought to be the best option. In our case, the patient's bone metastasis was discovered at a very late stage. It is reasonable to argue that this patient's disease may have been detected earlier by screening process; however, as far as prostate cancer screening is concerned there are no unified guidelines. The U.S. Preventive Services Task Force (USPSTF) used to recommend against PSA-based screening for prostate cancer [7]. Very recently the USPSTF backtracked on previous recommendation and suggested the PSA based screening is an 'individual decision'. On the other hand, the American Urological Association (AUA) panel recommends shared decision-making for men aged 55-69 years that are considering prostate cancer screening. The greatest benefit of screening appears to be in men ages 55-69 years. The USPSTF Guidelines, AUA, American College of Physicians (ACP), American Cancer Society (ACS) and the Canadian Task Force on the Periodic Health Examination all stress the importance of informed decision making. Unfortunately, our patient did not follow up with any physician precluding his opportunity to have those important discussions.

MM is a cytogenetically heterogeneous clonal plasma cell proliferative disorder [8] and is almost always preceded by an asymptomatic premalignant stage termed monoclonal gammopathy of undetermined significance (MGUS) [9]. The International Myeloma Working Group Criteria defines MM as clonal bone marrow plasma cells more than $10 \%$ or biopsy-proven extramedullary bony or plasmacytoma and either a myeloma defining event (evidence of end organ damage such as hypercalcemia, renal insufficiency, anemia and bone lesions, commonly abbreviated as CRAB) or any biomarkers of malignancy (clonal bone marrow plasma cell percentage more than $60 \%$, uninvolved serum free light chain ratio $>100$ and more than one focal lesion on MRI studies). Clinical presentation of MM may overlap with that of advanced prostatic cancer with bony metastasis. In our case, the patient did fulfill the CRAB criteria, which is one of the diagnostic features of MM; however, we could not make a definitive diagnosis without a bone marrow biopsy.

\section{Conclusions}

This case report sheds light on the importance of preventative care in routine outpatient care, which can often screen, identify, and detect malignancies at earlier stages. It also signifies the importance of an interdisciplinary approach and precise knowledge in differentiating and diagnosing such malignancies. In our patient's case, his extensive bone disease precluded his ability to be weight bearing which is an uncommon finding only seen in extensive metastatic bone disease. A definitive diagnosis is warranted to guide appropriate therapy and management of all malignancies. This case also demonstrates the overlap between clinical features of MM and metastatic prostate cancer. Ultimately, patient preferences dictate the extent of diagnostic evaluation and management; therefore, it is of utmost importance to engage patients in their ongoing medical and preventative care.

\section{Additional Information \\ Disclosures}

Human subjects: Consent was obtained by all participants in this study. WCGME-IRB issued approval WCGME04202018MSP. IRB case number WCGME04202018MSP is considered exempt from IRB review as it is a case study. Conflicts of interest: In compliance with the ICMJE uniform disclosure form, all authors declare the following: Payment/services info: All authors have declared that no financial support was received from any organization for the submitted work. Financial relationships: All authors have declared that they have no financial relationships at present or within the previous three years with any organizations that might have an interest in the submitted work. Other relationships: All authors have declared that there are no other relationships or activities that could appear to have influenced the submitted work.

\section{References}

1. Merrild EH, Baerentzen S, Bouchelouche K, Buus S: Vertebral myeloma mimicking prostatic carcinoma metastasis in 68 Ga PSMA PET/CT. Clin Nucl Med. 2017, 42:790-92. 10.1097/RLU.0000000000001773

2. Florimonte L, Orunesu E, Castellani M, Longari V, Cortelezzi A: 18F Choline PET/CT positive lytic bone lesions in prostate cancer and accidental myeloma detection. Clin Nucl Med. 2016, 41:394-96. 10.1097/RLU.0000000000001113

3. Segamwenge IL, Mgori NK, Yussuf SA, Mukulu CN, Nakangombe P, Ngalyuka PK, Kidaaga F: Cancer of the prostate presenting with diffuse osteolytic metastatic bone lesions: a case report. J Med Case Rep. 2012, 6:425. 10.1186/1752-1947-6-425

4. Rajendiran G, Green L, Chhabra G: A rare presentation of prostate cancer with diffuse osteolytic metastases and PSA of 7242 ng/ml. Int J Case Rep Imag. 2011, 2:16-20. 10.5348/ijcri-2011-09-55-CR-5

5. Loblaw A, Virgo K, Nam R, et al.: Initial hormonal management of androgen-sensitive metastatic, recurrent, or progressive prostate cancer: 2007 update of an American Society of Clinical Oncology practice guideline. J Clin Oncol. 2007, 25:1596-1605. 10.1200/JCO.2006.10.1949

6. Heyns CF, Basson J, Van der Merwe A, Zarrabi AD: Clinical (non-histological) diagnosis of advanced prostate cancer: evaluation of treatment outcome after androgen deprivation therapy. S Afr J Surg. 2014, 52:82-85.

7. Moyer VA: Screening for prostate cancer: U.S. Preventive Services Task Force recommendation statement . 


\section{Cureus}

Ann Intern Med. 2012, 157:120-34.

8. Palumbo A, Anderson K: Multiple myeloma. N Engl J Med. 2011, 364:1046-60. 10.1056/NEJMra1011442

9. Landgren O, Kyle RA, Pfeiffer RM, et al.: Monoclonal gammopathy of undetermined significance (MGUS) consistently precedes multiple myeloma: a prospective study. Blood. 2009, 113:5412-17. 10.1182/blood2008-12-194241 